\title{
Attenuation of flood waves in part-full pipes
}

\author{
by \\ P. Ackers, M.Sc.(Eng.), A.M.I.C.E., A.M.I.Mun.E. \\ and
}

A. J. M. Harrison, B.Sc., Dip.H.E.(Delft.), A.M.I.C.E.

Mr L. H. Watkins (Principal Scientific Officer, Road Research Laboratory, Department of Scientific and Industrial Research) wrote that the experiments reported by Ackers and Harrison had provided very useful knowledge of the behaviour of flood waves in partly filled pipes, and marked another stage in the rapid advance now being made into a more scientific approach to the problems of surface water drainage. The Authors did not comment on what impact they thought their experiments might have on current development of new methods of designing surface water sewers, in particular the R.R.L. Hydrograph method. It could be inferred from $\S 3$ of the Paper that a recorded retension curve was essential when the R.R.L. Hydrograph method was employed in the examination of an existing system. This is not so; with the aid of a digital computer the approximate retention relation can be readily calculated.

40. To assess the relative accuracy of the Authors' method of calculation and of the reservoir retention technique used in the R.R.L. Hydrograph method the trapezoidal input hydrograph shown in Fig. 2 has been routed through pipe retention by the R.R.L. method, for the four positions along the pipe illustrated. The values of maximum instantaneous depth then obtained were compared in Table 1 with the values given by the characteristics solution.

Table 1: Maximum instantaneous Depth

\begin{tabular}{c|c|c}
\hline \multirow{2}{*}{$\begin{array}{c}\text { Distance along pipe } \\
(f t)\end{array}$} & \multicolumn{2}{|c}{ Maximum instantaneous depth $(f t)$} \\
\cline { 2 - 3 } & $\begin{array}{c}\text { Characteristics } \\
\text { solution }\end{array}$ & $\begin{array}{c}\text { R.R.L. Hydrograph } \\
\text { method }\end{array}$ \\
\hline 28.4 & 0.43 & 0.49 \\
255.7 & 0.36 & 0.41 \\
483.0 & 0.33 & 0.32 \\
710.2 & 0.32 & 0.30 \\
\hline
\end{tabular}

41. The agreement between the two methods was remarkably good. It was problematical, however, whether the method of characteristics could be applied, possibly as modifications to the R.R.L. method, to the more complex case of a sewer

* Proc. Instn civ. Engrs, vol. 28, July 1964, pp. 361-382. 
network supplied with water at variable rates along its length, and whether such application would result in significant improvement in accuracy. More research would be required to establish this.

42. The Authors might care to consider, as a possible practical application of this method, the fairly common case of a long length of sewer forming part of a network but with no run-off entering along its length. The R.R.L. method gave in this case a gradual decrease in sewer size, and it could be seen from Table 1 that the characteristics method was likely to give a similar design. However, if the Authors could produce a practicable method of carrying out such a calculation it would be very useful to compare the two methods in a few actual design cases.

Dr E. J. Sarjinson (Department of Civil Engineering, University of Sheffield) observed that two cases of flood wave attenuation occurred in pipes. In the problem considered by the Authors, the flood water was introduced into the conduit at one point. Alternatively, the flood water could be led into the conduit at frequent intervals. This occurred in a sewer fed by gullies and branches, and the Authors had referred to methods of calculating the effects of attenuation on p. 362 .

44. The Authors had shown that the attenuation of a single wave in a conduit could be expressed in terms of two dimensionless parameters, both involving the volume of water in the wave. The profile of the wave had little or no influence on its attenuation. Dr Sarginson had found ${ }^{\theta}$ that the discharge from a pipe fed at frequent intervals by storm water was little affected by the shape of the hydrograph provided that the duration of the storm and the total precipitation were known. He had further shown ${ }^{18}$ that the discharge due to a particular storm was little affected by the size of the conduit, so long as it did not surcharge. As the Authors had pointed out (p. 362), the flood hydrograph produced by a rainstorm was modified by overland flow as well as by temporary storage or attenuation in the conduit. It was reasonable to conclude that drainage calculations could be carried out with sufficient accuracy by assuming a hydrograph of simple shape (e.g. a rectangle). Attenuation in the two cases could then be allowed for respectively by the Authors' dimensionless parameters, and by Watkins' assumption of a constant proportional depth of flow.

Dr D. M. McDowell (Consultant) did not agree that the Authors' basic equations (1) and (2) did not provide the required framework within which the results could be plotted. They could be put into a rational dimensionless form which would show very clearly the appropriate parameters to plot. The method adopted by the Authors was to use dimensionless parameters chosen to illustrate the effect of selected variables such as the wave height and distance along the pipe. This method was widely used but unless the parameters were properly chosen there was a risk that the plotted results would conceal as much as they revealed. A fundamental failing was that the plotted results gave no indication whatever of scale. The experimental results plotted in Figs 3-11 could equally well represent waves $1 \mathrm{~cm}$ or $10 \mathrm{~m}$ in height. It would be shown that there was, in fact, a scale effect that was concealed by the empirical choice of dimensionless parameters. The Authors' conclusion ( 837 ) that because the graphs were dimensionless in form, they were representative of storm sewers of all diameters and floods of all durations and magnitudes was therefore incorrect.

46. The Authors selected the parameters $h / D$ and $x D^{2} / V$ for their first trial plots. Their introduction of the wave volume $V$ was an interesting idea which had brought out one of the main features of the behaviour of the laboratory waves. They then obtained more consistent graphs by including all the data for a given base flow in a plot of $h / D$ against $x D^{2} / V \div Q_{\mathrm{f}} / \sqrt{g D^{5}}$ where $Q_{\mathrm{f}}$ was the pipe-full discharge. The Authors pointed out in $\& 26$ that the parameter $Q_{t} / \sqrt{g D^{5}}$ was proportional to the Froude number; this was not correct. It was constant for each slope, whereas the Froude number varied with each base flow and wave depth. 
47. The parameter $Q_{\mathrm{r}} / \sqrt{g D^{5}}$ could nevertheless be made to yield more useful information. Putting $Q_{t}=v_{f} \pi D^{2} / 4$ where $v_{t}$ was the mean velocity of flow when the pipe was just full, and using one of the forms of the pipe friction formulae:

$$
v_{f}=C \sqrt{m s}=\sqrt{2 g D s / f}
$$

where $C$ was the Chézy coefficient, $m$ the hydraulic mean depth, and $f$ the universal friction coefficient, lead to:

$$
Q_{\mathrm{r}} / \sqrt{g \overline{D^{5}}}=\pi C \sqrt{s} / 8 \sqrt{g}=\pi \sqrt{s / 8 f}
$$

48. It could now be seen that the sorting of results which the Authors obtained by use of the parameter $Q_{\mathbf{f}} / \sqrt{g D^{5}}$ gave the same effect as the use of $C \sqrt{s}$ or $\sqrt{s / f}$. The coefficients $C$ and $f$ both depended on the Reynolds number of the flow and therefore depended on temperature, unless the flow fell within the rough turbulent flow range, in which case they would be constant for a given ratio of relative roughness. The Reynolds number $R$ could be estimated for the pipe-full case, using assumed values for kinematic viscosity $\nu$ and for $f$.

Reynolds number $R=v_{\mathrm{t}} D / \nu$ and $v_{\mathrm{f}}=\sqrt{2 g D S / f}$. Assuming that $\nu=0.000012 \mathrm{sq}$. $\mathrm{ft} / \mathrm{sec}$ (water at $60^{\circ} \mathrm{F}$ ) and $f=0.030, R$ could be calculated for the pipe diameter $D=0.264 \mathrm{ft}$ and the corresponding values of the friction factor $f$ could then be found from published curves:

$\begin{array}{lcc}\text { Pipe slope } S & 1 / 50 & 1 / 1000 \\ \text { Pipe-full Reynolds No. } R & 26300 & 5900 \\ \text { Friction factor } f & 0.024 & 0.035\end{array}$

These values of Reynolds No. fell well within the range of smooth turbulent flow.

49. If the experimental results reproduced in Figs 3-10 were examined, it would be seen that the scatter of the results at each slope was rather less than that due to the results for all slopes taken together; e.g. the results for slope $1 / 50$ fell consistently above the mean curve in Fig. 4 , but below it in Fig. 6 . Could the Authors say whether these fluctuations were due to variations of Reynolds number between the experiments?

50. The Authors did use the equations of motion (1) and (2) (\$5) in an attempt to calculate the discharge $Q_{p}$ corresponding to maximum depth. To obtain a solution, the Authors used values of $\partial Q / \partial t$ based on the mean value of the rate of increase of discharge during the rising part of the flood wave. This was not admissible; the equations could only describe conditions at a particular time and place. The value of $\partial Q / \partial t$ corresponding to the instant of maximum depth must be used. This would be small and might well be of the same order as $d h / d x$ which the Authors neglected. In fact, $Q_{p}$ was not likely to differ from $Q_{n}$ by more than a few per cent. The difference would be greatest for rapidly varying flows such as would occur at the steeper slopes, but in any case would be small because, at the time of maximum depth, accelerations would be nearly zero. Fig. 11 showed just the reverse; the steepest slopes showed values of $Q_{\mathrm{p}} / Q_{\mathrm{n}}$ near to, but consistently less than, unity, while the two mildest slopes showed values of $Q_{\mathrm{p}}$ going up to $200 \% Q_{\mathrm{n}}$. In fact, the plotted results for each slope showed little correlation between $Q_{\mathrm{p}} / Q_{\mathrm{n}}$ and the Authors'

$$
\frac{1}{A g S} \frac{\partial Q}{\partial t}
$$

51. There was an alternative rational approach to this problem that would have overcome all the difficulties that had arisen. The solution lay in the Authors' equation (2), the well-known equation of continuity. In order to solve this equation it was necessary to know the pipe geometry, initial conditions before the onset of the flood wave and variations of water level with time at a few points along the pipe. The equation was best used in the form $\frac{\partial Q}{\partial x}+B \frac{\partial h}{\partial t}=0$ which was identical with the Authors' equation (2). $Q$ was the instantaneous value of discharge at time $t$. Know- 
ledge of $B \frac{\partial h}{\partial t}$ at any point enabled $\frac{\partial Q}{\partial x}$ to be computed at that point. Knowledge of the initial conditions of flow then enabled $Q$ to be calculated at any time by a process of finite differences. There was a fair amount of labour involved but it was not excessive, the channel geometry being particularly simple. In any case, it could be reduced by use of a digital computer. Cumulative errors could be detected and eliminated because the flow reverted to base flow after passage of the wave. It was not necessary to measure any discharge other than the base flow and the instrumental difficulties mentioned in $\$ 30$ did not arise.

52. When the variations of discharge had been found at a few points, it would become possible to evaluate all the terms of the dynamic equation, equation (1), with the exception of the friction term $g i$. Thus the effect of friction could be found. The ratios of the terms to each other during the passage of the wave could then be calculated and their variation between the different experiments could be found. When this had been done, the factors that played an important part in the propagation of the wave could be selected for further study. Dr McDowell suggested that this study of the equations of motion, which could easily be put in a rational dimensionless form ${ }^{17}$ would lead to better analysis of experimental results through proper selection of dimensionless parameters. He also suggested that any dimensionless plot should include a clear statement of the range of dimensions that were covered by the data.

Mr K. V. H. Smith (Lecturer in Civil Engineering, Southampton University) wrote that the solution of problems of unsteady part-full flow in pipes, or any form of open channel, was complex when means other than hydrologic routing had to be applied. The method of characteristics offered a means of solution, but was time-consuming. For major works, however, a finite difference method using a computer could be applied. ${ }^{18}$

54. Considered from the viewpoint of the routine design of stormwater drainage systems something simpler was required and the Authors were to be congratulated on bringing the variables together in suitable dimensionless form, thus enabling the construction of Figs 3-10. For general use, though, it would seem essential to extend the curves in Figs 3 and 4 in the direction of $D / h$ approaching 1.0 and it would presumably be necessary to run further experiments in order to accomplish this.

55. In certain circumstances, as in a long outfall, the curve could be used to determine the decay of a flood peak with progress along the sewer. The integration of the method into a comprehensive system of storm-water sewer design, however, must call for a great deal more research work. As far as Great Britain was concerned considerable development work would be required, leading to a revision of the Road Research Laboratory Method as far as the modification of the initial hydrograph was concerned.

56. It was instructive to investigate storm flows from one or two sewered areas, to determine the order of magnitude of peak flow reduction involved. For this purpose Mr Smith had considered two recorded storm-water system outflow hydrographs given in Road Research Technical Paper No. 55. ${ }^{2}$

57. The first was from Oxhey (Area 1), from the storm of 5 November, 1955. This was from a relatively small area and the peak discharge was 3.35 cusec. The volume of the wave $V$, measured from the hydrograph, was $1860 \mathrm{cu}$. $\mathrm{ft}$. There was no base flow. Let it be supposed that downstream of the outfall point there was a $1000 \mathrm{ft}$ length of pipe before any further connexion was made.

58. In investigating an area in which Oxhey (Area 1) was a contributory area it would be necessary to consider the flow in the $1000 \mathrm{ft}$ length of pipe flowing part full. For example, an 18-in. dia. pipe at a gradient of 1 in 520 would discharge 3.35 cusec when running $0.6 \mathrm{D}$ full. $Q_{\text {f }}$, the pipe-full discharge, was $5.0 \mathrm{cusec}$, then

$$
\frac{x \sqrt{g D^{9}}}{V Q_{r}}=\frac{1000 \sqrt{g \times 1 \cdot 5^{9}}}{1860 \times 5 \cdot 0}=3.78
$$


Assuming no base flow, Fig. 3 of the Paper indicated a reduction in proportional depth to 0.52D. Assuming the hydraulic grade line parallel to the invert, this gave $Q_{n}=2.67$ cusec.

In order to assess the effect of the unsteady flow condition, an estimate of the value of $\frac{\partial Q}{\partial t}$ at the downstream point from the known hydrograph of flow at the upstream point could be derived. This gave $\frac{\partial Q}{\partial t}=0 \cdot 01$.

Then

$$
\frac{1}{A g S} \cdot \frac{\partial Q}{\partial t}=\frac{1}{0.93 \times 32 \cdot 2} \times \frac{520}{1} \times 0.01=0.17
$$

From Fig. 11 of the Paper $\frac{Q_{\mathrm{p}}}{Q_{\mathrm{n}}}=1.06$. Hence the actual flow $1000 \mathrm{ft}$ downstream of the outfall point was $2.67 \times 1.06=2.83$ cusec.

The reduction in peak flow in this case was from 3.35 cusec to 2.83 cusec, or $15.5 \%$.

59. It was interesting to compare the reduction in peak flow due to different sizes of conveyance system for the same flow. Mr Smith considered the recorded outflow hydrograph from Oxhey (Area 11) due to the storm of 5 June, 1954. The peak discharge was 200 cusec and the volume of the wave $V$ could be measured off as 290000 cu. $\mathrm{ft}$. Let it be supposed that downstream of the outfall point there was a $2000 \mathrm{ft}$ length of pipe before any major connexion was made.

(i) Suppose that the discharge was taken in a 72-in. dia. pipe at a gradient of 1 in 300 , flowing $0 \cdot 65 \mathrm{D}$ full. Then $Q_{\mathrm{f}}=264 \mathrm{cusec}$.

$$
\frac{x \sqrt{g D^{9}}}{V Q_{1}}=\frac{2000 \sqrt{g \times 6^{9}}}{290000 \times 264}=0.38 .
$$

Assuming no base flow, it was clear from Fig. 3 of the Paper that little diminution in peak flow would occur.

(ii) Alternatively, consider that the discharge was taken in two 72-in. dia. pipes at a gradient of 1 in 1200, flowing 0.65D full. Then for each pipe $V$ was halved and $Q_{1}$ was halved. Hence for each pipe:

$$
\frac{x \sqrt{g D^{9}}}{V Q_{i}}=1 \cdot 48 .
$$

From Fig. 3 of the Paper it could be estimated that the depth of flow would reduce from $0.65 \mathrm{D}$ to $0.6 \mathrm{D}$. This gave $Q_{\mathrm{n}}$ for each pipe equal to 88.5 cusec.

From the inflow hydrograph, $\frac{\partial Q}{\partial t}$ for the downstream point could be estimated at 0.03 for each pipe. $\frac{Q_{\mathrm{p}}}{Q_{n}}$ was approximately 1.02 so that the peak flow at the lower end of the $200 \mathrm{ft}$ of twin 72 -in. dia. pipe would be approximately $2 \times 88.5 \times 1.02$, or 180 cusec (compared with 200 cusec at the upstream end). As was to be expected the system with the greater storage capacity and lower velocities had the greater effect in reducing the peak.

Mr J. A. Hall (Senior Assistant Engineer, Borough Engineer's Department, Ilford) wrote to ask for the Authors' comments on the following points.

61. Could the results of the Paper be applied to pipelines having changes of diameter and gradient, and how were such changes to be dealt with?

62. If changes of diameter and gradient could be dealt with, it might be possible to reduce pipe sizes downstream; there was, no doubt, in any particular case a limit to the possible reduction. Would the Authors please show briefly how this might be done and how the limit might be arrived at. 
63. Where values of $h / D$ fell outside the limits of the experimental results, to what extent could extrapolation of the Author's curves be relied on? Would it be preferable to use equation (3) where this was applicable?

Mr F. V. Appleby (Chartered Civil Engineer) congratulated the Authors, both for the pioneer nature of their experiment and for carrying out the lengthy and tedious work of producing a characteristic solution of equations (1) and (2) in direct relationship to an experiment. He said that the last differential coefficient in equation (1) should be $d v / d x$. Apart from some diagrammatic illustrations ${ }^{10}$ there seemed to exist no earlier attempt at direct comparison such as was given in Fig. 2. It was a pity that the Authors did not complete the picture by calculating and measuring velocities. The order in which the maximum of velocity, discharge rate and depth occurred was rightly emphasized and had an important bearing on the argument in $\S 32$, although surprisingly enough the Authors made no reference to Fig. 2 at that time. In $\S 23$ the Authors had discussed the question of the $x$ co-ordinate which had to be resolved if the work was to be applied to practical conditions. The Authors did not satisfactorily resolve the question. In $\S 22$ they referred to the influence of previous history in the formation of the hydrograph-and suggested that volume rather than 'shape' dominated the attenuation process. In $\$ 26$ they had drawn attention to the composite nature of the distance parameter which could be stated as the product of a length $\left(V / D^{2}\right)$ weighted with the Froude number $(F)$. $D^{2}$ was proportional to the area demand $\left(A_{0}\right)$ of inflow which was attenuated to a value $A_{1}$ for example when a trunk sewer of length $l$ was added to the system. $V / A_{0}$ was a length $\bar{x}$, relating to this input; this was very much a factor of 'shape' (or distribution) and set a linear scale for the whole subsequent process. This formation length $\bar{x}$ was a characteristic of the drainage basin as a whole and defined a virtual origin upstream of the section of interest. ${ }^{19}$ This concept was necessary to resolve the evident difficulty referred to in $\$ 23$. The length of the trunk main could then be considered in terms of the product relating to input. Since continuity of volume was supposed, and bearing in mind that $\vec{x}$ was a mean length, an attenuation equation could be derived as follows,

$$
A_{0} \bar{x} F_{0}=A_{1}\left(\bar{x} F_{0}+l / 2\right)
$$

or

$$
A_{1} / A_{0}=1 /\left(1+l / 2 F_{0} \bar{x}\right)
$$

Using some numerical values supplied by the Authors, Mr Appleby found that this formula followed fairly closely, and certainly consistently, a sequence of actual results for no base flow conditions. The estimation of the Froude number was a difficulty. The Authors' use of pipe full flow $Q_{r}$ was open to question since the free surface was rapidly approaching zero. A closer fit with actual results could have been obtained if the $F$ values were somewhat larger in all cases.

65. For the reasons given in $\S 32$ this could well be so. Would the Authors please clarify this important question in the interests of a more general application of their work. Since $h / D$ was approximately proportional to $\sqrt{ } \vec{a} / \vec{A}$ the attenuation curve of Fig. 3 was closely followed by the formula

$$
h / D=0.50 / \sqrt{(1+l / 10)}
$$

by setting the zero point as unity and changing the scale to one tenth of the values shown. A rectangular channel would attenuate the flow to an even smaller amount in the same distance. For Fig. 3 the effective values of $2 F_{0} \bar{x}$ was ten units and the attenuation at section 25 refered to a trunk main distance of the order of $2 \frac{1}{2}$ times the input parameter. $\bar{x}$ was a measure of the size of the input drainage network and $F_{0}$ the hydraulic characteristics of its arterial system. For a small town the value of $\bar{x}$ could be of the order $5000 \mathrm{ft}$ and if $F_{0}$ was near unity for example, then at the scale figure of 25 of Fig. 3 the result was a trunk outfall of the length order of $25000 \mathrm{ft}$ ! In their experimental set-up the flood wave was generated in the trunk main itself. 
Could the Authors suggest a mode of modification when the value of $F$ for the flood was very different from that of the channel?

66. Mr Appleby said he had commented on the question of a practical zero for the distance co-ordinate at some length in view of its obvious importance and the Authors' lead in regard to a suitable parameter. In $\S 26$ the solidus $(/)$ was missing in the Froude No. reference. References to full pipe flow and diameter $D$ became meaningless when any attempt was made to think of the results in general terms of open channel flow and it would be helpful if the Authors would comment on the possible generalization of their parameters. Some practical suggestion from the Authors in regard to the base flow factor would be of value to those concerned with combined sewerage systems. It was interesting to note that the time parameter was similar to that commonly used by hydrologists i.e. $V / Q_{\mathrm{f}}=K$ say: a response time for the input system. Inplicit in the statements about diameter $D$ was the diameter required by an input flow and the Authors' statement in $\S 28$ of the independence of the time parameter and the hydraulic factors did not seem to be correct. The important distinction in $\$ \$ 31,32$, between actual discharge and 'normal' discharge was well demonstrated. The coefficient of $\partial h / \partial x$ in equation (8) should be $Q^{2} D / A^{3}$. At this point of maximum depth of flow the value of $\partial Q / \partial t$ was governed by $A \partial v / \partial t$ only and since the derivative of $A$ changed sign near its peak, it followed that the corresponding value of $\partial Q / \partial t$ was a maximum and was therefore the point of inflexion of the $Q$ hydrograph. The value of actual discharge $\left(Q_{\mathrm{n}}\right.$ nearly) at this point was always less than the real peak value and the proportion by which one exceeded the other could be seen by reference to Fig. 2 . In the case of the pairs of $Q$ and $h$ hydrographs shown, the ratios were $1: 30 ; 1: 15 ; 1: 10$. The values of $\partial Q / \partial t$ on the rising and falling limbs were very different in practice and were by no means equivalent as suggested by the Authors in devising equation (12). The argument that followed was interesting but inconclusive. The value of $\partial Q / \partial t$ at maximum depth depended on a rate of change of velocity which was so rarely measured through a flood. This Paper was full of new and stimulating material and it was hoped that the Authors would continue the study.

Professor F. M. Henderson (Professor of Civil Engineering, University of Canterbury, Christchurch, New Zealand) congratulated the Authors on their comprehensive experimental study, which had brought to light some interesting subsidence properties possessed by the sharply peaked fast-rising flood waves characteristic of stormwater drainage systems.

68. Undoubtedly, the results of most substantial interest were embodied in the dimensionless number $x \sqrt{\left(g D^{9}\right) /\left(V \bar{Q}_{\mathrm{f}}\right)}$ (Figs 3-6), and it was of some interest to attempt a theoretical justification for the form of this number, in particular for the part played by the wave volume $V$ and the bed slope $s$, the latter being implied in the pipe-full discharge $Q_{r}$.

69. Previous work by Professor Henderson ${ }^{20}$ had to some extent elucidated the effect of wave form on subsidence by distinguishing between the 'river crest' A (Fig. 12), at which $\partial h / \partial x=0$, and the 'local crest' $B$, at which $\partial h / \partial t=0$. The latter corresponded to the crests of the curves drawn on the $h-t$ plane in Fig. 2. From equation (2) it followed that $\partial Q / \partial x=0$ at $B$, and it could also be shown that $\mathrm{B}$ was always downstream of $\mathrm{A}$. From the total differential equation

$$
\frac{d h}{d x}=\frac{\partial h}{\partial x}+\frac{\partial h}{\partial t} \cdot \frac{d t}{d x}
$$

if followed that

$$
\frac{d h}{d x}=\frac{\partial h}{\partial x}
$$




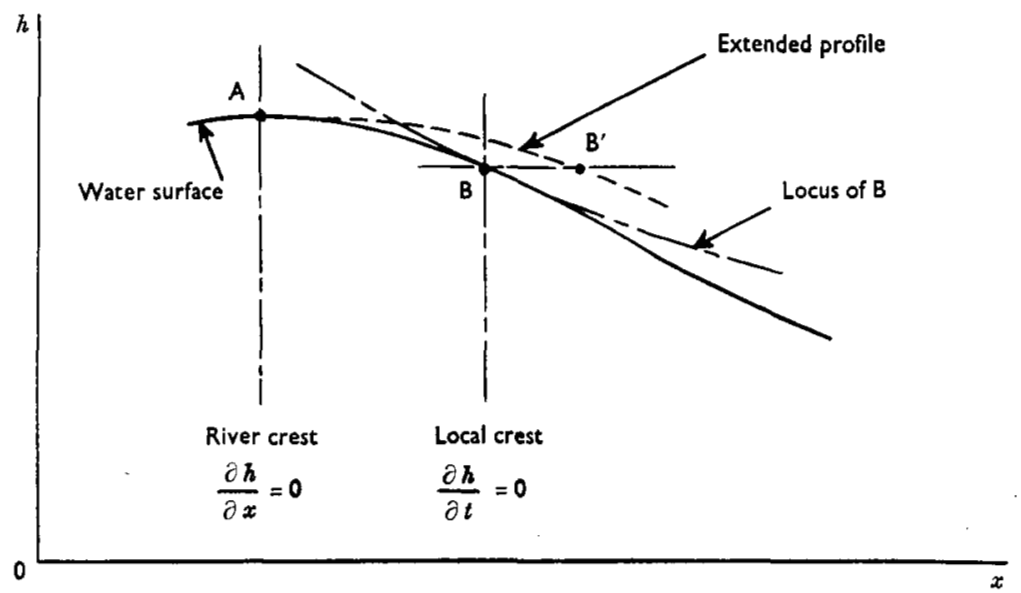

Fig. 12: INSTANTANEOUS FloOd WAVE PROFILE

at $B$, i.e. that the locus of $B$ on successive positions of the wave profile had the same slope as the profile itself at $\mathbf{B}$.

70. The effect of variation in the wave volume $V$ could now be studied by considering a second profile produced by longitudinal extension of the first, as in Fig. 12 . $B$ moved to $B^{\prime}$, and the question was whether $B^{\prime}$ would also be the local crest of the new profile. It could be shown that it was exactly true for the 'mild' or slow-rising flood waves treated by Professor Henderson, ${ }^{20}$ and approximately true for the sharplypeaked waves studied by the Authors. If followed that $d h / d x$, the rate of crest subsidence with distance, reduced in direct proportion to the factor by which the profile was longitudinally extended. Since the wave volume $V$ increased in direct proportion to that factor, the inclusion of $V$ in the denominator of the number $x \sqrt{ }\left(g D^{9}\right) /\left(V Q_{\mathrm{f}}\right)$ was justified, provided that the waves under consideration were of approximately similar shape and varying volume (as was presumably the case in the Authors' study).

71. The inclusion of the term $Q_{\mathrm{f}}$ in the denominator was less easy to justify. Since $Q_{f} \alpha s^{\frac{1}{2}}$, the effect was that the rate of attenuation with distance of a wave of specified size and shape increased with the square root of the slope. On the other hand, Professor Henderson's study of slow-rising waves indicated that the distance $\mathrm{AB}$ in Fig. 12 was inversely proportional to the slope, i.e. that the attenuation rate $d h / d x$ increased directly with the slope. Further study seemed to be required in order to bridge the gap between the two results.

72. Because only one pipe-size was used, the experimental support for the exponent of $D$ in $x \sqrt{\left(g D^{9}\right) /\left(V Q_{\mathrm{f}}\right)}$ was not conclusive, although the scaling operation on which Fig. 2 was based offered some indirect support. Dimensional considerations also required that $D$ should have the exponent $9 / 2$, provided that $V$ and $Q_{\mathrm{f}}$ were correctly placed. It would have been interesting, however, to place the matter beyond doubt by experiments on other sizes of pipe.

73. Comparison of equation (11) with the well-known Jones formula

$$
\frac{Q_{\mathrm{p}}}{Q_{\mathrm{n}}}=\sqrt{\left(1-\frac{1}{s V_{\mathrm{w}}} \frac{\partial h}{\partial t}\right)} \quad . \quad . \quad . \quad . \quad .
$$

led to a curious result. If the approximations on which equation (11) was based were generally applicable, then equation (11) should in the limit be applicable to the 
same situation, i.e. long mild flood waves of low Froude number, to which the Jones formula was applicable. In that case the two formulae would be equivalent, i.e.

$$
\frac{1}{A g} \frac{\partial Q}{\partial t}=\frac{1}{V_{\mathrm{w}}} \frac{\partial h}{\partial t}
$$

74. For a mild wave the quotient of those two derivatives was simply equal to $d Q / d h$; thence by equation (3)

$$
\frac{B v_{\mathrm{w}}}{A g}=\frac{1}{v_{\mathrm{w}}}
$$

i.e.

$$
v_{\mathrm{w}}=\sqrt{(\overline{A g / B)}}=v_{\mathrm{c}}
$$

the critical velocity. It appeared therefore that equation (11) could be equivalent to the Jones formula only if the wave velocity equalled the critical velocity, so that the approximations leading to equation (11) were valid only for the fast-rising sharplypeaked waves studied by the Authors.

75. For the reasons given in $\$ \S 71$ and 73 , some of the results appeared to be valid only for the particular type of wave studied by the Authors. It was therefore to be hoped that their useful and thought-provoking study would lead to further work which would extend and generalize the results so far obtained.

The Authors, in reply, said that they were very pleased with the number of contributions to the discussion of the Paper. They were grateful to Mr Appleby for pointing out some typographical errors in the text. Some others which they had noticed were:

(i) the left-hand side of equation (11) should read $\frac{Q_{\mathrm{p}}}{Q_{\mathrm{n}}}$,

(ii) the equation on Fig. 11 should be equation (11) and the numbers on the $x$-axis should be negative.

77. Several points in the discussion had been raised by more than one contributor. The Authors said that they would deal with these comments subject by subject, rather than reply point by point to each contributor.

78. The statement that the parameter $Q_{f} / \sqrt{\left(g D^{5}\right)}$ was proportional to the Froude number was challenged by McDowell and Appleby. The Froude number was essentially the ratio of the gravitational to the inertial forces in a fluid system and for non-steady flow conditions a typical value could be defined from steady uniform flow at a representative depth. Thus it was pointed out that the Froude number of a half-full pipe was $2.035 Q_{1} / \sqrt{\left(g D^{5}\right)}$. It was perhaps not well enough known that for a given slope the Froude number in a pipe was nearly constant over the range of proportional depth of 0.1 to 0.7 as illustrated in Fig. 13 in which the ratio Froude number to $Q_{t} / \sqrt{g} D^{5}$ was plotted against proportional depth. The relation was derived as follows:

The Froude number, $F$, was given by

$$
F=Q / \sqrt{g A^{3} / B}
$$

where $Q, A$, and $B$ were the 'normal' discharge, cross-sectional area and surface width respectively corresponding to depth $d$. It followed that

$$
\frac{F}{Q_{f} / \sqrt{\left(g D^{5}\right)}}=Q_{\mathrm{P}} /\left(\frac{64}{\pi^{3}} \frac{B_{\mathrm{P}}}{A^{3}{ }_{\mathrm{P}}}\right)
$$

where $A_{\mathrm{P}}=A /\left(\pi D^{2} / 4\right), B_{\mathrm{P}}=B / D$ and both are a function only of the proportional 


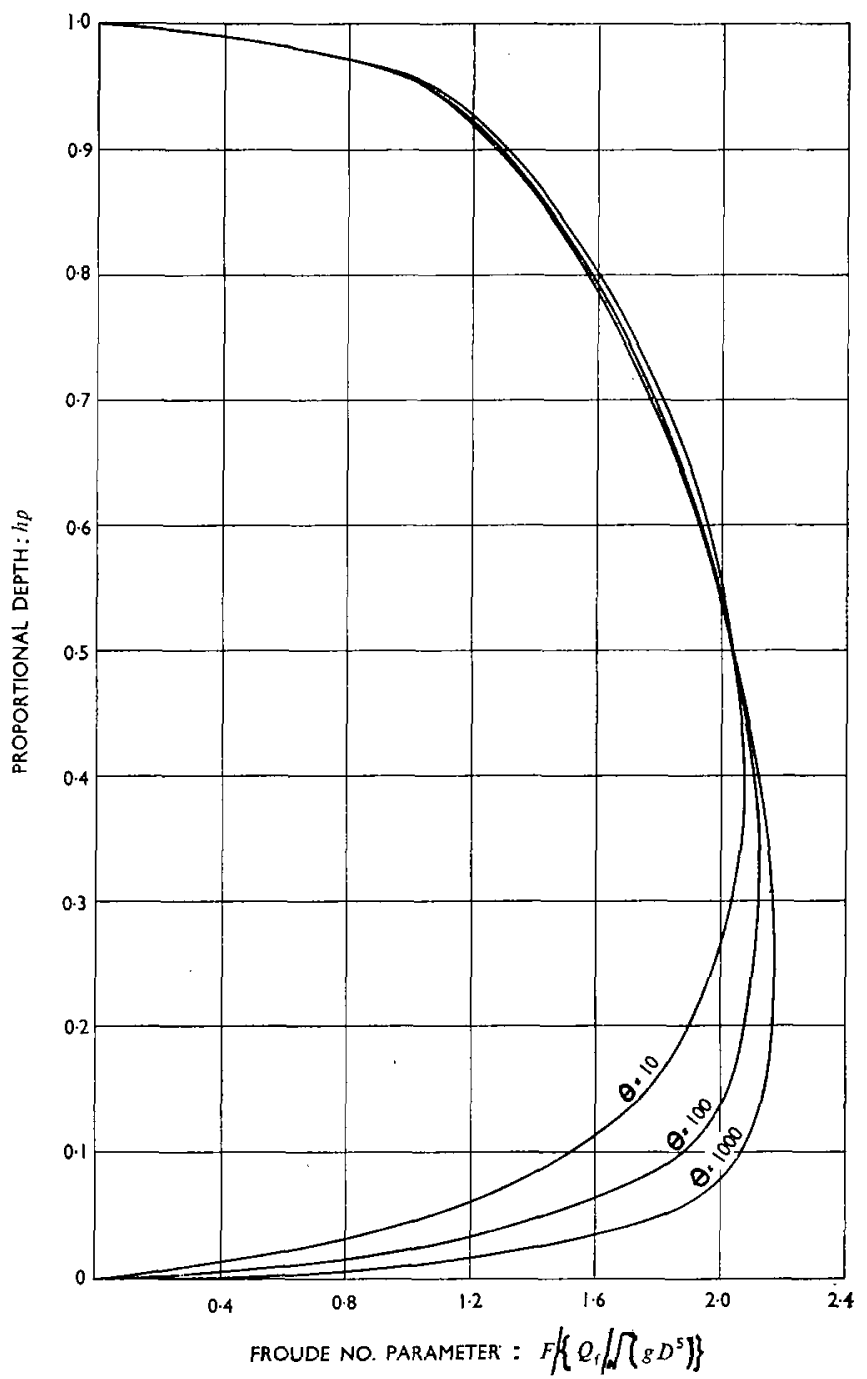

FIG. 13

depth $h_{\mathrm{P}}=h / D .^{21}$ The proportional discharge, $Q_{\mathrm{P}}=Q / Q_{\mathrm{f}}$ and is a function of $h_{\mathrm{P}}$ and $\theta$ where

$$
\theta \simeq\left(\frac{k}{D}+\frac{1}{1100 D s^{1 / 3}}\right)^{-1} \quad \text {. . . . . . . }
$$

79. Both Henderson and McDowell questioned the form of the distance parameter $\left(x g^{1 / 2} D^{9 / 2}\right) /\left(V Q_{i}\right)$ against which the proportional height of the wave peak was plotted. It was agreed that the term $Q_{\mathrm{f}}$ in the denominator implied that the rate of attenuation 
was proportional to $l / s^{1 / 2}$ whereas Henderson's paper ${ }^{20}$ showed that it was proportional to $l / s$. However his result applied only to channels with low values of Froude number while in the present case the Froude number (at half-full depth) was in the range 0.4 to 2 . There was little doubt that the index $n=1$ for the parameter $\left\{Q_{1} / \sqrt{\left(g D^{5}\right)}\right\}^{n}$ gave the best fit for the data as a whole. As McDowell pointed out, where the trend for a given slope was above the mean line for one depth of base flow it was below the mean line at another, and hence it was unlikely that a revision of $n$ would improve the correlation. It was not thought that these fluctuations were due to variations of Reynolds number between the experiments since for any one slope the variation of Reynolds number was the same for tests at all depths of base flow.

80. The scale relationships between two geometrically similar systems governed by equations (1) and (2) could be quite simply deduced. Each of the terms of the equation had to bear the same relationship to the others in both model and prototype Thus writing the length, time, velocity, and hydraulic gradient scales as $\lambda_{\mathrm{L}}, \lambda_{\mathrm{T}}, \lambda_{\mathrm{V}}, \lambda_{\mathrm{I}}$ and noting that $g$ and $s$ were common to both there followed,

From (1)

From (2)

$$
\frac{\lambda_{\mathrm{L}}}{\lambda_{\mathrm{L}}}=\frac{\lambda_{\mathrm{V}}}{\lambda_{\mathrm{T}}}=\frac{\lambda_{\mathrm{V}}^{2}}{\lambda_{\mathrm{L}}}=\lambda_{\mathrm{I}}=1
$$

$$
\lambda_{\mathrm{V}} \lambda_{\mathrm{L}}=\lambda_{\mathrm{L}}{ }^{2} \lambda_{\mathrm{T}}=\lambda_{\mathrm{L}} \lambda_{\mathrm{V}}
$$

Hence $\lambda_{\mathrm{V}}=\lambda_{\mathrm{T}}=\lambda_{\mathrm{L}}{ }^{1 / 2}$ and $\lambda_{\mathrm{L}}=1$. These were the well-known relationships for a Froude scale model. McDowell showed that the Froude number parameter could be written as $\sqrt{(s / 8 f)}$ where the friction factor $f=2 g m i / v^{2}$. Then to obtain similarity $\lambda_{\mathrm{f}}=\lambda_{\mathrm{L}} \lambda_{\mathrm{I}} / \lambda_{\mathrm{v}}{ }^{2}=1$. Thus the experimental pipe was a Froude-scale model of any larger prototype pipe so long as $f$ was the same in model and prototype. McDowell's comment, 'A fundamental failing was that the plotted results gave no indication whatever of scale', indicated a failure to appreciate the merits of dimensionless plotting. The results applied to any scale or system of units so long as $\lambda$ was the same, or alternatively, so long as $Q_{\mathrm{f}}$ was scaled up in accordance with the Froudian scale relationships.

81. For example, at a slope of $1: 1000, Q_{\mathrm{f}}$ in the experimental pipe of $0 \cdot 264 \mathrm{ft}$ diameter was 0.042 cusec. For a pipe of $3 \mathrm{ft}$ diameter the scaled up discharge was $0.042 \times(3 / 0.264)^{5 / 2}=18.3$ cusec. Consulting the tables in reference 21 the corresponding roughness for the slope, diameter, and discharge specified was $0.01 \mathrm{ft}$, a typical value for a slimed sewer. It was believed, however, that the results of the experiments would apply to pipes having other values of the friction factor; for a given slope and diameter a greater rate of attenuation would be expected with greater roughness and the graph also indicated this through the reduction in $Q_{\mathbf{r}}$. The point could not be proved conclusively, however, without further experiments with the pipe roughened or alternatively with a larger pipe of the same surface roughness, as suggested by Henderson.

82. In $\$ 31$ it was pointed out that the discharge at a given depth in non-steady flow could not be assumed equal to the normal discharge, $Q_{n}$, at that depth. Conversely, for a given maximum input discharge, $Q_{\mathfrak{p}}$, the initial maximum depth was not necessarily the same as the normal depth for that discharge. Fig. 11 was an attempt to correlate $Q_{\mathfrak{p}} / Q_{\mathrm{n}}$ with a known characteristic of the input wave. Thus given $Q_{\mathrm{p}}$ and $\partial Q / \partial t$ for an incoming wave the figure could be used to determine the peak depth through a calculation for $Q_{\mathrm{n}}$. It was evident from a study of Fig. 2 that at a given position the peak discharge $Q=Q_{\mathrm{p}}$ (when $\partial Q / \partial t=0$ ) occurred slightly ahead of the wave crest when $h=h_{\mathrm{p}}$ at $\partial h / \partial t=0$. Applying equation (11) strictly, both $Q$ and $Q_{\mathrm{n}}$ applied to the instant when $\partial h / \partial t=0$ and $Q$ was therefore somewhat less than the assumed value $Q_{p}$. Furthermore $\partial Q / \partial t$ was the slope of the discharge-time curve at that instant and not the average values used in the plotted points of Fig. 11. These points were made in $\$ 32$ and 33 of the Paper, but were repeated in view of Appleby's 
and McDowell's comments. The assumptions had to be made in order to plot the experimental results. The Authors agreed with Henderson that equation (11) should only be used for conditions similar to those in the experiments; the Jones formula was only applicable if the Froude number was low.

83. In fact the points for the 1:1000 slope agreed well with the theoretical line; those for steeper slopes fell generally below the curve but all showed a rising trend. The following semi-empirical equation was a better fit than equation (11) to the experimental points,

$$
\frac{Q_{\mathrm{p}}}{Q_{\mathrm{n}}}=\sqrt{\left(1-\frac{\Delta Q / \Delta t}{1000 A g s^{2}}\right)} \quad . \quad . \quad . \quad . \quad .
$$

where $\Delta Q / \Delta t$ was the slope of the discharge-time curve defined by equation (12).

84. In $\S 50 \mathrm{McD}$ owell suggested that $Q_{\mathrm{p}}$ would not differ from $Q_{\mathrm{n}}$ by more than a few per cent, and that the difference would be greatest at the steepest slopes. The

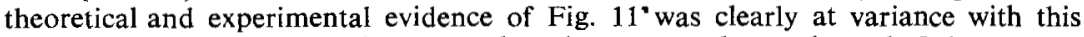
point of view. It was true, however, that the steeper slopes showed $Q_{p} / Q_{n}$ values less than unity. Apart from the reasons suggested in $\$ 33$ there were the following possible explanations,

(a) at a slope of 1:250 flow was critical and stationary waves formed at the depth probes. It was probable that this lead to overestimates of peak depth and hence of $Q_{n}$.

(b) At slopes of 1:50 and 1:100 flow was supercritical, In these circumstances the depth at the entrance to the pipe was controlled by the discharge and the entrance geometry only and was probably close to the critical depth. It was likely, therefore, that the depths recorded at the first probe unit were greater than the normal depths for this reason.

85. Appleby showed in $\S 66$ that in the characteristics solution, Fig. 2, the discharge at the peak of depth was considerably less than the maximum discharge. Nevertheless, if the maximum discharge was determined from the equation $Q_{\mathrm{p}}=$ $\left(Q_{\mathrm{p}} / Q_{\mathrm{n}}\right) \times Q_{\mathrm{n}}$ where $Q_{\mathrm{p}} / Q_{\mathrm{n}}$ was determined from equation (11) taking $\delta Q / \delta t$ as the mean slope of the front of the wave on the discharge-time plot and $Q_{\mathrm{n}}$ was the normal discharge corresponding to the maximum depth, then the values obtained were $0.604,0.414$, and 0.327 cusec for $x=28.4,255 \cdot 7$, and $483.0 \mathrm{ft}$ respectively. These agreed closely with the values $0.609,0.423$, and 0.322 cusec calculated by the method of characteristics and plotted on Fig. 2. It scemed, therefore, that the overestimate in the assumed value of $\partial Q / \partial t$ counterbalanced the underestimate involved in the assumption in equation (11) that $Q=Q_{\mathrm{p}}$ at $\partial h / \partial t=0$, and lead to a reasonable estimate of the peak discharge at least for slopes of $1 / 1000$.

86. Appleby suggested in $\S 66$ that the point of maximum depth coincided with the point of inflexion on the $Q$ hydrograph. A careful study of Fig. 2 indicated that this was not so, as was also shown by differentiation of the continuity equation. From $Q=\Delta v$ it followed that

$$
\frac{\partial}{\partial t}\left(\frac{\partial Q}{\partial t}\right)=\frac{\partial^{2} Q}{\partial t^{2}}=A \frac{\partial^{3} v}{\partial t^{2}}+2 \frac{\partial A}{\partial t} \frac{\partial v}{\partial t}+\frac{\partial^{3} A}{\partial t^{2}}
$$

At $\frac{\partial h}{\partial t}=0, \frac{\partial A}{\partial t}=0$ and

$$
\frac{\partial^{2} Q}{\partial t^{2}}=A \frac{\partial^{2} v}{\partial t^{2}}+v \frac{\partial^{2} A}{\partial t^{2}} \neq 0
$$

87. In $\$ 51 \mathrm{McD}$ owell suggested that the discharge at any point in the pipe could have been calculated by a finite differences method from the continuity equation. The method was in fact considered during the analysis of the data but proved impracticable because of the spacing of the depth probes. Fig. 2 illustrated this point; 
at any instant the wave was not recorded at more than three positions, giving only three values of $\delta Q / \delta x$ from which a $Q$ vs. $x$ curve could not be determined.

88. Hall and Smith asked how the curves of Figs 3-6 could be extended towards $h / D=1$. It was agreed that some further experiments would be required to extend the curves accurately, but in practice it was unlikely that the curves would be used for $h / D$ greater than about 0.82 since at this proportional depth the steady uniform discharge was equal to the pipe-full discharge. 'The following equation which had been found to fit all the data of Figs 3-6 should apply also to high values of $h / D$,

$$
\frac{h}{D}-\frac{h_{0}}{D}=1 /\left(2+\frac{x \sqrt{g D^{9}}}{15 V Q_{\mathrm{r}}}\right)^{1+1 \cdot 5 h_{0} / D}
$$

Equation (24) could be expected to hold for all values of $h_{0} / D$ between 0 and 0.5 .

89. Several contributors were interested in the direct application of the results of the Paper to the design of a length of pipe without connexions. The method proposed by the Authors was as follows:

(a) with $D, s$ and $Q_{\text {p }}$ given, calculate the maximum depth and maximum area, $A$, at the inlet assuming steady uniform flow conditions;

(b) from the mean rate of rise of discharge, $\Delta Q / \Delta t$, and the calculated value of $A$, determine $Q_{\mathrm{p}} / Q_{\mathrm{n}}$ from Fig. 11 or equation (22), hence obtain $Q_{\mathrm{n}}$;

(c) from $Q_{\mathrm{n}}$, calculate the maximum depth and area again;

(d) if the values are significantly different from the original calculations, repeat steps (b) and (c) until a satisfactory approximation is achieved;

(e) enter the appropriate attenuation curve at the relevant $h / D$ value for the inlet and move to the right along the curve by $\frac{l \sqrt{g D^{9}}}{V Q_{I}}$ where $l$ is the length of pipe; read off the new value of $h / D$ for the outlet; alternatively equation (24) could be used for this calculation;

(f) calculate the new value of $Q_{\mathrm{n}}$ from the new value of $h$. If necessary the nonsteady correction can be applied to determine $Q_{p}$ from Fig. 11 using an estimated value of $\Delta Q / \Delta t$.

90. The Authors were most grateful to Smith for presenting some worked examples as space did not permit them to do so in the Paper. The examples were very useful and illustrated the order of change in peak discharge to be expected in practical cases. However it would be seen from the preceding paragraph that a small error had been made in the examples in the way in which the non-steady flow effect was allowed.

91. The method above could be applied to pipes having changes of gradient and slope. The discharge, depth, and $\Delta Q / \Delta t$ should be calculated as described at the downstream end of each length of pipe of given gradient and diameter. The figures should then be used to calculate the peak depth at the upstream end of the next section of pipe. The wave could then be routed through that section, and so on.

92. It was certainly true that for a given gradient the pipe sizes could be reduced downstream; however there was no limit to the possible reduction as suggested by Hall, except that the peak discharge could not, of course, fall below the base-flow discharge.

93. Appleby suggested that there was a question of the $x$-parameter which had to be resolved if the work was to be applied to practical conditions. In fact the Authors did not think there was a problem here. The origin of the $x$-parameter was of no importance-all that was required was the value of the $x$-parameter at the upstream end of the length of pipe being considered, which was determined automatically on entering the attenuation curve at the relevant $h / D$ value.

94. The Authors did not fully understand how Appleby arrived at the first equation in $\S 64$. It seemed that he was equating the initial volume of the wave $\left(=A_{0} \bar{x}\right)$ to the volume of the attenuated wave after the peak had travelled a distance $l$ assuming that the profile was triangular and had a base length of $2 \bar{x}+l / F_{0}$. The 
equation in $\S 65$ appeared to fit Fig. 3 fairly well if $l$ was replaced by $x \sqrt{g D^{9}} / V Q_{\mathrm{r}}$ but in this case the Authors could not see how it followed from the second equation of $\S 64$. $F_{0} \bar{x}$ was proportional to $Q_{\mathrm{r}} V$ and was by no means constant; data on Fig. 3 had values of $Q_{\mathrm{f}} V$ varying between 0.8 and 0.03 .

95. Fig. 14 showed a logarithmic plot of the experimental data of Fig. 3. The fit of Appleby's equation was nearly as good as equation (24) but the latter was to be preferred because of its more general nature.

96. Watkins' Table 1 gave an interesting comparison between the complete solution of the hydrodynamic equations by the method of characteristics and the much simpler, but less academically justifiable, flood routing technique used by hydrologists. The agreement appeared to be fairly good but there was some irregularity in the decay function of the R.R.L. solution. The objection to applying this method (or that of Sarginson) to flood routing through a pipe without connexions was that it was based on the assumption that the depth was constant throughout the pipe at any one time. Fig. 2 showed that this was certainly not the case in the Authors' experiments. Furthermore the methods lead to the conclusion that the attenuation of a wave was a function only of the ratio of the storage volume to the

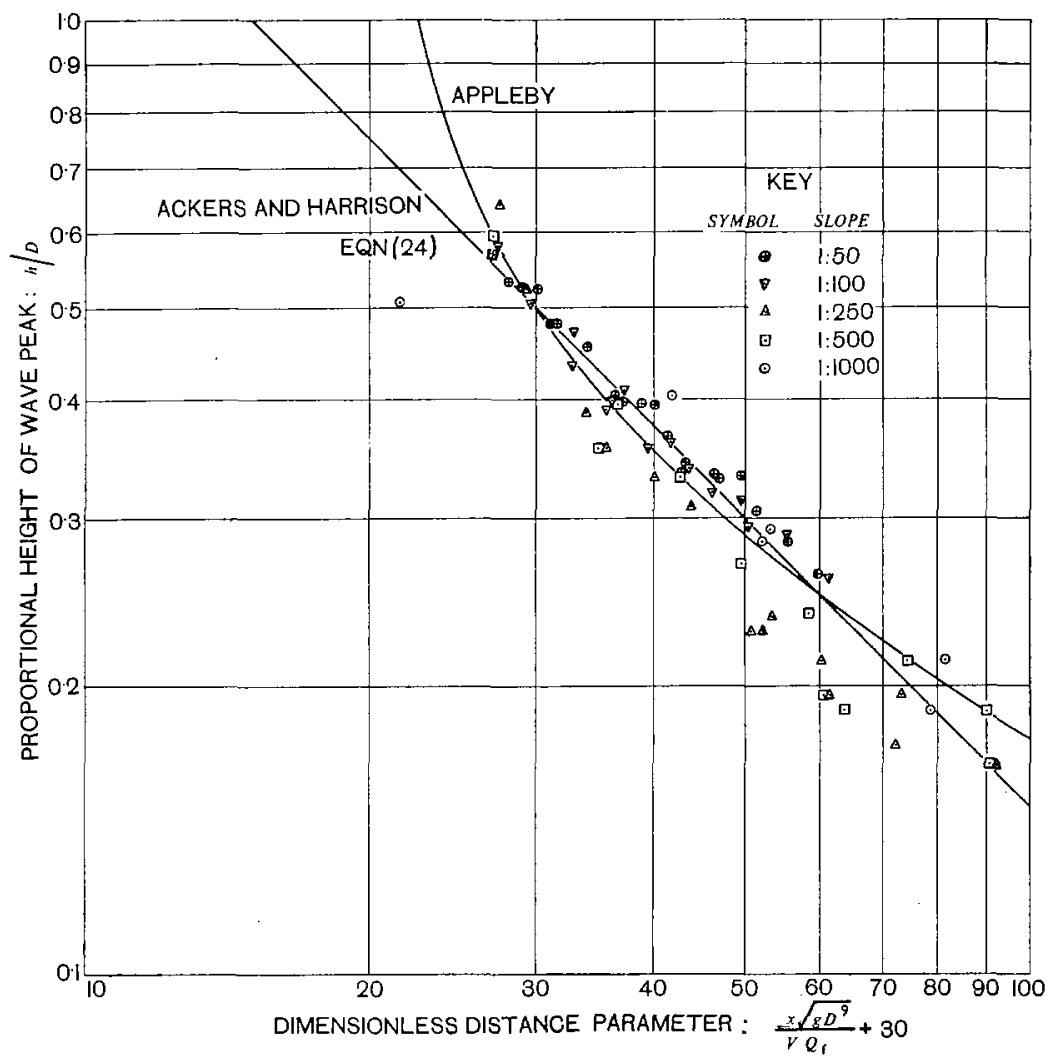

Fig. 14: LOGARITHMIC PLOT OF EXPERIMENTAĹ DATA IN FIG. 3 
wave volume ${ }^{22}$ whereas the Authors' experiments and Henderson's ${ }^{20}$ theoretical study show that slope (or Froude number) was also a factor.

97. It was not the Authors' object to put forward a general design method for sewers. However, it was hoped that the results presented on the simpler case of attenuation in a single pipe would have some direct application and also would assist the development of methods for dealing with the more complex case of a sewerage network.

\section{REFERENCES}

16. Sarginson E. J. Contribution to discussion on paper by Watkins (Ref. 3). Proc. Instn civ. Engrs, 1964, 27 (March), 605.

17. BIRkoff G. Hydrodynamics, a study in logic, fact and similitude. Princeton, 1950.

18. Isaacson E., Stoker J. J. and Troesch A. Numerical solution of flow problems in rivers. Proc. Am. Soc. civ. Engrs, 1958, HY5, 84 (Oct.), 1810.

19. Appleby F. V. Run-off dynamics, a heat conduction analogue of storage flow in channel networks. International Association of Scientific Hydrology, Publication No. 38, vol. 3, Rome, 1954, 338-348.

20. Henderson F. M. Flood waves in prismatic channels. Proc. Am. Soc. civ. Engrs, 1963, HY4, 89 (July), 39-67.

21. Ackers $P$. Tables for the hydraulic design of storm-drains, sewers and pipelines. Hydraulics Research Paper 4, H.M.S.O., London, 1963.

22. Harrison A. J. M. Contribution to discussion on Paper by Watkins (Ref. 3). Proc. Instn civ. Engrs, 1964, 27 (March), 592-596. 